\title{
NONSTEROIDAL ANTI-INFLAMMATORY DRUGS AS THERAPEUTIC ALLIES OF THE GUT MICROBIOME ON CHRONIC INFLAMMATION
}

\author{
Fabian Mermans, Evelien Heiremans, Maud Van Belleghem, Axelle Meersschaut, Emma Hernandez-Sanabria \\ Center for Microbial Ecology and Technology (CMET), Ghent University, Ghent, Belgium
}

\begin{abstract}
Our gut harbours around $10^{14}$ bacteria of more than 1000 species, accounting for approximately $2 \mathrm{~kg}$ of biomass. The gut microbiome plays several vital functions in processes such as the development of the immune system, food digestion and protection against pathogens. For these functions to be beneficial for both host and microbiome, interactions are tightly regulated. Gut and immune cells continuously interact to distinguish among commensal microbiota, harmless foodstuff, and pathogens. A fine balance between inflammatory and anti-inflammatory state is fundamental to protect intestinal homeostasis. Nonsteroidal anti-inflammatories (NSAIDs) are a class of drugs used for management of pain and inflammation. These compounds have heterologous structures but similar therapeutic activities. The target of all NSAIDs are the isoforms of cyclooxygenase enzymes (COX): the primarily constitutive form COX-1, and the inducible from COX-2. Both isoforms catalyse the conversion of arachidonic acid to PGH2, the immediate substrate for specific prostaglandin and thromboxane synthesis. The gut microbiota plays a role in drug metabolism, resulting in altered bioavailability of these compounds. Additionally, complex host-microbiome interactions lead to modified xenobiotic metabolism and altered expression of genes involved in drug metabolism. These effects can be at gut tissuelevel, or distant, including in the liver. Besides the gut microbiome influencing drug metabolism, drugs also impact the microbial communities in the gut. As different drugs exert selective pressures on the gut microbiome, understanding this bidirectional relationship is crucial for developing effective therapies for managing chronic inflammation.
\end{abstract}

Key words: gut microbiome, non-steroidal anti-inflammatories, inflammation, dysbiosis.

\section{Introduction}

The gut microbiota includes more than 100 trillion bacterial cells [1], which can be divided into six dominant phyla: Firmicutes, Bacteroidetes and Actinobacteria, and the less abundant Proteobacteria, Fusobacteria and Verrucomicrobia [2]. The human microbiome is not only composed of collective genomes from bacteria, but also from those of archaea, viruses, and eukaryotes colonizing the gut [3]. The composition of the microbiota will depend on environmental factors, diet, age and host genetics [4]. Inter- and intra-individual differences are significantly prevalent, although several species such as Faecalibacterium prausnitzii, Roseburia intestinalis and Bacteriodes uniformis have been consistently identified in large scale screenings. The gut microbiome transforms indigestible substrates from food, such as xyloglucans, into short chain fatty acids, which can be used as energy source for the colonocytes [5]. These metabolic activities may directly or indirectly impact health and disease in the host. The commensal microbiota can train the immune system to induce regulatory $\mathrm{T}$ cells, which then prompt tolerance to these bacteria [6].

Correspondence to: Emma Hernandez-Sanabria, Ph.D.

VIB - Center for Microbiology, KU Leuven Laboratory of Molecular Bacteriology, Rega Institute, Herestraat 49 - Bus 1028, 3000 Leuven, Belgium

Phone: +32163722 22

E-mail: emma.hernandezsanabria@kuleuven.vib.be

Received December 22 $2^{\text {nd }}, 2019$
In this way, commensal-induced immunity contributes to maintain mucosal tolerance and protect the host from disease [7]. Such exchanges are tightly regulated: gut and immune cells continuously interact to distinguish between commensal microbiota, harmless foodstuff, and pathogens. A fine balance between inflammatory and anti-inflammatory status is needed to preserve intestinal homeostasis [8].

Interest in developing colon-targeted drug delivery systems has increased in recent years [9]. These formulations are intended for gastrointestinal-related diseases, such as colorectal cancer (CRC) and inflammatory bowel disease (IBD). Despite the numerous research in this field, there are still questions that need to be answered, as differences in microbiome composition may ultimately impact drug metabolism between subjects [10]. For example, which characteristics of the microbiome contribute to the pharmacokinetic differences between individuals? Does the region in the colon reflect a specific colonization pattern, which will later trigger metabolic differences? Do long-term alterations in the gut microbiome generate metabolic dysbiosis? If so, to what extent? Gaining insight into the gut metabolic potency is essential, because characterization of the players involved in drug disposition in the gut will contribute to ensure optimal drug efficacy and safety profile.

Existing colon-specific drug delivery systems hold some limitations. Besides the microbial influence on 
drug bioavailability and pharmacokinetics, low solubility in presence of bile acids and the small aqueous volume in the colon may hinder drug efficacy [11]. Moreover, understanding drug transporter activity is fundamental to improve drug passive permeability. Current models to predict drug efficacy consider plasma concentrations to describe the pharmacokinetics and pharmacodynamics. However, drugs designed for targeting the gut undergo an extensive first pass metabolic clearance. This implies that the relation between plasma concentrations and efficacy has a low predictive value. For this reason, investigating microbiome-drug-host interactions towards improving ADME and safety responses of intestinal targets is fundamental [12].

\section{Xenobiotic Metabolism of the Gut Microbiome}

Chemotherapeutic outcomes are mainly linked to human genetic polymorphisms [13], but the impact of the human gut microbiome has been overlooked, as it has been even called the "forgotten organ". Although microbiome research has flourished in recent years, the association between the microbiota and xenobiotics metabolism remains underexplored. The host cytochrome CYP2C9 predominantly eliminates lipophilic drugs through the liver, whereas orally administered drugs encounter the gut microbiota before reaching host tissues [10]. This first-pass metabolism by the colonic microbiota must be considered, as the metabolites generated from this process can impact drug activity, resulting in toxicity for the host and further inflammation, or altering gut microbiota and producing dysbiosis [14].

The anaerobic environment of the human gut prevents the microbial use of oxygen as terminal electron acceptor for oxidation $[10,14]$. Thus, anaerobic respiration and increase in microbial growth are facilitated by the two main metabolic transformations: reduction provides alternative electron acceptors, while hydrolysis ensures substrates that can be used by microorganisms [15]. Since these two reaction types are commonly observed, core microbial species or core gene families performing such functions are hypothesized to participate [16].

Links between gut bacteria and drug metabolism are via direct and indirect mechanisms. Direct mechanisms involve the transformation of the drug into metabolites with a different effect. Conversely, indirect mechanisms are those influencing drug transport or metabolism [14]. Knowledge of these metabolic pathways is fundamental to predict whether drug metabolism and disposal will be impacted.

Additionally, biliary excretion is essential for drug recirculation and ultimate metabolism. In this respect, $\beta$ glucuronidases can play a key role, because they are important for host drug detoxification [17]. Glucuronic acid is coupled to several substrates in the liver, thereby increasing molecular weight and solubility. Consequently, elimination through urine or faeces is en- hanced, and gut bacteria can reactivate these products, generating higher toxicity. Likewise, non-steroidal antiinflammatory drugs (NSAIDs) have side-effects [18]. Microbial $\beta$-glucuronidases can separate the aglycone from the glucuronide, so the aglycone is absorbed into the enterocytes and transformed into reactive metabolites, harming the mitochondria and the endoplasmic reticulum. In this way, not only the host detoxification pathway is diminished, but also inflammation is evoked as a result of decreased mucosal integrity [19]. The impact of the gut microbiota on hepatic drug-processing genes is enzyme-specific and age and sex-dependent, with patterns varying throughout life span and developmental periods, and xenobiotic pathways significantly downregulated in male mice at 90 days of age [20].

Moreover, probiotic interventions have confirmed that microbiome manipulations not involving antibiotics can influence the expression of hepatic drug-processing genes during adulthood [21]. Poorly absorbed antibiotics such as vancomycin and rifaximin, may indirectly modulate hepatic CYP gene expression, as they bypass hepatic metabolism and impact gut bacteria [22]. Understanding the outcomes of indirect host-microbiomedrug interactions occurring because of drug use, consumption of dietary supplements, or environmental toxins should be considered in drug development, safety pharmacology, and pharmacokinetic profiling [20].

\section{The Gut Microbiome and Xenobiotic Metabolism during Chronic Inflammation}

Although research efforts are ongoing to more precisely define a healthy gut microbiome [23], one of the most frequently reported findings across an array of disorders is a narrowing of gut microbiome diversity often accompanied by more specific but less consistent compositional alterations at various taxonomic levels. Dysbiosis refers to the state where the composition of the microbiome is disrupted [24, 25], providing continuous immunological stimulation and leading to anomalies in the immune response. The polarized induction of immune cells and the subsequent increased amounts of pro-inflammatory $\mathrm{T}$ cells may fuel the development and severity of the disease. Excessive inflammation results in loss of epithelial integrity, which in turn leads to further bacterial translocation and thus further induction of inflammation $[8,24]$. This condition is observed in a number of gastrointestinal diseases such as inflammatory bowel disease (IBD) and colorectal cancer (CRC) [26], central nervous system disorders such as depression and schizophrenia [27], obesity [28], diabetes mellitus [29], cardiovascular disorders [30], rheumatoid arthritis [31] and multiple sclerosis [24], and asthma and atopy in children [32]. Research found that Faecalibacterium prausnitzii is reduced in patients with ulcerative colitis, another chronic inflammatory condition of the colon [33]. This bacterium has been reported to promote the accumulation of regulatory $\mathrm{T}$ cells $\left(\mathrm{T}_{\text {reg }}\right)$, which contribute to anti-inflammatory responses [34]. On the 
contrary, Enterobacteriaceae, Bacteroides, Clostridium ramosum and Akkermansia muciniphila are increasingly present in patients with IBD. These organisms have also been linked with increased inflammation [35]. Although the importance of the gut microbiome in chronic inflammatory conditions has been reported [36], it is still unclear whether dysbiosis is a consequence or a cause of associated diseases [8, 24].

Gut microbiota are known to play a role in the metabolism of drugs, hereby having an effect on the host health. Many of the therapeutic interventions for chronic disorders are subject to biotransformation by the gut microbiome, and the functional implications of changes in gut bacterial communities for xenobiotic metabolism are yet to be described. The gut microbiome can affect drug therapy via direct or indirect mechanisms. The direct mechanisms compromise metabolization of the drugs by the microbiota, resulting in altered bioavailability of these compounds. Indirect mechanisms include complex host-microbiome interactions, resulting in a modified xenobiotic metabolism of the host [10]. In the latter case, one of the routes is that microbiota affect host gene expression involved in drug metabolism. These effects can be either local, meaning in the gut tissue [37], or distant, including in the liver [38].

Understanding how alterations in gut microbiota profiles influence host response to chemotherapeutic drugs may have important clinical implications. Stratifying patients based on their gut microbiome composition may assist in identifying responders and non-responders to immunotherapy [39] for the treatment of epithelial tumours and melanomas [20, 40]. Other mechanisms modulated by the gut microbiome include the translocation and immunomodulation following interventions with cyclophosphamide, doxorubicin, and anti-CLTA-4 therapies [41].

\section{Dynamics of Nonsteroidal Anti-Inflammatory Drugs (NSAIDs) towards the Gut Microbiome}

Nonsteroidal anti-inflammatory drugs (NSAIDs) are a class of drugs used for inflammation management. The compounds in this group are heterologous in structure but show similar therapeutic activity [42]. The target of all NSAIDs are cyclooxygenase enzymes (COX). There are two isoforms of COX, the primarily constitutive form COX-1, and the inducible from COX-2 [42]. COX-1 and COX-2 both catalyse the conversion of arachidonic acid to $\mathrm{PGH}_{2}$ [43]. $\mathrm{PGH}_{2}$ is the immediate substrate for some cell specific prostaglandin and thromboxane synthases [42].

COX-1 is expressed in different tissues such as the kidneys, lungs, stomach, ileum and colon [44]. It is considered to be a "housekeeping" enzyme, playing an important role in the production of prostaglandins that serve homeostatic functions [45]. These prostaglandins, among others, maintain the integrity of the gastric mucosa, mediate normal platelet function and regulate re- nal blood flow [46]. COX-2 on the other hand, is normally not expressed in tissues with the exception of the macula densa of the brain and kidney, the placenta, the vessel walls and the heart, where it is believed to play a role in homeostatic functions [47]. This isoform of the enzyme is upregulated in response to tissue damage or the presence of pro-inflammatory cytokines [43]. In turn, COX-2 is responsible for the generation of proinflammatory prostaglandins, thus contributing to inflammation [45]. One of these prostaglandins is $\mathrm{PGE}_{2}$, which is implicated in carcinogenesis. COX-2 expression is found to be increased in colorectal cancer causing proliferation, enhanced angiogenesis and suppression [48] of apoptosis (Pugh \& Thomas, 1994; Surh et al., 2001).Thus, COX-2 is the main target of chronic inflammation therapy using non-steroidal anti-inflammatory drugs (Fig. 1).

\begin{tabular}{l} 
Inhibition \\
undesirable \\
Homeostatic functions \\
$\begin{array}{l}\text { Gl tract } \\
\text { Renal function } \\
\text { Platelet function } \\
\text { Macrophage differentiation }\end{array}$ \\
\hline
\end{tabular}

Fig. 1 Isoforms of COX enzymes and their functions

Orally administered drugs are subject to the so called "first-pass metabolism", where the gut microbiome plays a crucial part. Prior to reaching the target tissue, microbiota and liver metabolize the drugs, altering their bioactivity [10]. The bacterial metabolism results in three scenarios, namely activation, inactivation, and increased toxicity of the drug. In the first situation, the pharmaceutical compound is activated into its therapeutic window, resulting in increased drug efficacy. An example is the described transformation of sulindac to its bioactive sulphide metabolite. In the case of inactivation, the availability of the active drug is lowered resulting in decrease or loss of therapeutic effect. For instance, microbial arylamine $\mathrm{N}$-acetyltransferases inactivate the bioactive component of the anti-inflammatory drug sulfasalazine [14], while the NSAID diclofenac can exert toxic effects after being glucuronidated in the liver by the host and then being exposed to microbial $\beta$-glucoronidases in the gut $[10,18]$. In these scenarios, activity of the gut microbiome results in increased toxicity and adverse effects for the host. In addition, simultaneous use of probiotics significantly increased the microbiota-mediated enzymatic degradation of the antipyretic and analgesic paracetamol [20,39]. Similarly, short-term administration of a probiotic cocktail of L. acidophilus, B. lactis and Streptococcus salivarius to rats significantly increased azoreductase activity in $e x$ vivo incubation of sulfasalazine with colon contents, ultimately impacting the metabolism of this drug [49]. 
Besides the gut microbiome influencing drug metabolism, drugs also impact the microbial communities in the gut. As discussed earlier, a shift in gut microbiome, and more specifically dysbiosis, is linked to disease. Therefore, understanding the effects of drugs on the gut microbiota is crucial for providing suitable therapies [14]. Obvious examples include the use of antibiotics, resulting in altered gut microbiome [50]. NSAIDs have been reported to impact the gut microbiome and changes in composition have been observed [51] depending on the NSAID administered. Subjects exposed to aspirin showed greater abundance in Prevotella spp., Bacteroides spp., Barnesiella sp. and Ruminococcaceae. Celecoxib and ibuprofen users showed enrichment in Enterobacteriaceae and Acidaminococcaceae. Ibuprofen users were also enriched in Propionibacteriaceae, Pseudomonadaceae, Puniceicoccaceae and Rikenellaceae [52]. In contrast, a different study observed no effects on the microbiome composition after administration of celecoxib [53]. Sulfasalazine induced the expression of thioredoxins and nitrate reductases, while nizatidine, subject to bacterially mediated $\mathrm{N}$-oxide bond cleavage, up-regulated the expression of drug enzymes and transporters acting on nitrogen bonds [54]. This finding supports the earlier hypothesis that drugs may shift the microbiota to favour the abundance of taxa involved in its metabolism. Furthermore, this altered metabolic capacity of the microbiota could consequently affect not only the pharmacokinetics of subsequent doses of the drug itself (a phenomenon referred to as autoinduction) but also the pharmacokinetics of co-administered medication may be act as substrates of the same metabolic pathway or transporter. Production of diet-derived by-products such as like $p$ cresol (from protein digestion) can affect drug metabolism. Research showed that acetaminophen, an analgesic and antipyretic drug, underwent less sulfonation due to competitive O-sulfonation of $p$-cresol [55].

COX-1 plays a role in guaranteeing the mucosal integrity of the gastrointestinal tract and thus, non-specific NSAIDs blocking both isoforms of COX have been linked with intestinal damage [19]. These environmental changes place a selective pressure on the microbiome resulting in fluctuations in microbial composition [56]. Blocking intestinal bacterial enzymatic functions has been proposed to amend intestinal homeostasis, while enhancing efficacy and decreasing toxicity of IBD therapies, as realised for cancer therapies [4, 17]. Precision editing of the gut microbiota by tungstate ameliorates experimental gut inflammation through preventing the dysbiotic expansion of Enterobacteriaceae [57]. This is merely an example of the complex host-microbiomedrug interactions and it is likely that different drugs exert selective pressures on the gut microbiome through different mechanisms [14]. The use of designer lactic acid bacteria as factories for the production of antimicrobial and anti-inflammatory biomolecules may also have potential for the future treatment of infectious diseases, cancer, and metabolic diseases [54, 58].

\section{Conclusion}

It is clear that the gut microbiome play an enormous role in patient health, but also in pharmacokinetic and pharmacodynamic drug response. Before official approval, government agencies such as the FDA run three phases of clinical trials, where pharmacokinetics, pharmacodynamics, and safety of a drug are determined. However, gut bacteria is not currently considered within these evaluations, although the microbiome is the first checkpoint following drug intake. In vitro studies have shown that some NSAIDs can be metabolized by bacteria, potentially jeopardizing its anti-inflammatory effects. More than 60 interactions between drugs and the microbiome have already been documented. Unfortunately, most of the underlying molecular and genetic mechanisms remain unclear. The PharmacoMicrobiomics online database was launched in 2011 to assemble the literature about those interactions [59]. Future efforts may focus on combining available information about the drug-bug interactions on biochemical pathways with existing genomic, pharmacogenomic and human microbiome sequence databases [52].

Personalized medicine has achieved excellent results in some areas of medicine, such as oncology [4]. Yet, genetic factors can influence up to $50 \%$ of therapy responses [13]. It is at this crossroads that recent discoveries on the roles of gut microbiota have become paramount [60]. Stratification of patients based on their gut microbiome may improve treatment accuracy [17] and cost-effectiveness [61]. A combined strategy to restore eubiosis may require synchronised targeting of dominant pathobionts and replacing missing beneficial species or their functions by manipulating the bacterial microbiota with dietary strategies. Thus, homeostatic immune responses, and mucosal barrier function would be reconditioned. This integrated approach may result safer than the current lifelong treatments with immunosuppressive drugs, once remission has been accomplished by traditional therapies.

Understanding how our "second genome" [4] is involved in therapeutic responses could pave the way for approaches using the intestinal microbiome as the target for modulating drug efficacy and enabling tailored treatments. Revealing the specific mechanisms driving defective bacteria-host interactions will enable precision editing of gut microbiota functionality and composition for ameliorating chronic inflammatory diseases.

Acknowledgements. The authors acknowledge Dr. Racha El Hage and Dr. Ioanna Poulopoulou for the constructive discussions. This work was partially supported by a Postdoctoral Fellowship from Flanders Innovation and Entrepreneurship (Agentschap Innoveren \& Ondernemen). 


\section{References}

1. Valdes AM, Walter J, Segal E, Spector TD. Role of the gut microbiota in nutrition and health. BMJ 2018; 361:k2179.

2. Zhao L, Zhang X, Zuo T, Yu J. The composition of colonic commensal bacteria according to anatomical localization in colorectal cancer. Engineering 2017; 3:90-97.

3. Foster KR, Schluter J, Coyte KZ, Rakoff-Nahoum S. The evolution of the host microbiome as an ecosystem on a leash. Nature 2017; 548:43-51.

4. Zmora N, Zeevi D, Korem T, Segal E, Elinav E. Taking it personally: personalized utilization of the human microbiome in health and disease. Cell Host Microbe 2016; 19:12-20.

5. De Vuyst L, Leroy F. Cross-feeding during human colon fermentation. In: González-Ortiz G, Bedford MR, Bach Knudsen KE, Courtin CM, Classen HL, editors. The value of fibre: Engaging the second brain for animal nutrition. Wageningen Academic Publishers; 2019. pp 565-569.

6. Kato LM, Kawamoto S, Maruya M, Fagarasan S. The role of the adaptive immune system in regulation of gut microbiota. Immunol Rev 2014; 260:67-75.

7. Lathrop SK, Bloom SM, Rao SM, Nutsch K, Lio C-W, Santacruz N, Peterson DA, Stappenbeck TS, Hsieh C-S. Peripheral education of the immune system by colonic commensal microbiota. Nature $2011 ; 478: 250$

8. Shen $\mathrm{S}$, Wong $\mathrm{CH}$. Bugging inflammation: role of the gut microbiota. Clin Transl immunology 2016; 5:e72.

9. Amidon S, Brown JE, Dave VS. Colon-targeted oral drug delivery systems: design trends and approaches. Aaps PharmSciTech 2015; 16:731-741.

10. Spanogiannopoulos P, Bess EN, Carmody RN, Turnbaugh PJ. The microbial pharmacists within us: a metagenomic view of xenobiotic metabolism. Nat Rev Microbiol 2016; 14:273-87.

11. McConnell EL, Fadda HM, Basit AW. Gut instincts: explorations in intestinal physiology and drug delivery. Int J Pharm 2008; 364:213-226

12. Hernandez-Sanabria E, Heiremans E, Arroyo MC, Props R, Leclercq L, Snoeys J, et al. Short term supplementation of celecoxib shifted butyrate production on a simulated model of the gut microbial ecosystem and ameliorated in vitro inflammation. bioRxiv 2019; 679050 .

13. Tannock IF, Hickman JA. Limits to personalized cancer medicine. N Engl J Med 2016; 375:1289-1294.

14. Wilson ID, Nicholson JK. Gut microbiome interactions with drug metabolism, efficacy, and toxicity. Transl Res 2017; 179:204-222.

15. Arkhipova OV, Akumenko VK. Unsaturated organic acids as terminal electron acceptors for reductase chains of anaerobic bacteria. Microbiology 2005; 74:629-639. [In Russian]

16. Haiser HJ, Seim KL, Balskus EP, Turnbaugh PJ. Mechanistic insight into digoxin inactivation by Eggerthella lenta augments our understanding of its pharmacokinetics. Gut Microbes 2014; 5:233-238.

17. Kent DM, Steyerberg E, van Klaveren D. Personalized evidence based medicine: predictive approaches to heterogeneous treatment effects. BMJ 2018; 363:k4245.

18. Higuchi K, Umegaki E, Watanabe T, Yoda Y, Morita E, Murano $\mathrm{M}$, et al. Present status and strategy of NSAIDs-induced small bowel injury. J Gastroenterol 2009; 44:879-888.

19. Boelsterli UA, Redinbo MR, Saitta KS. Multiple NSAID-induced hits injure the small intestine: underlying mechanisms and novel strategies. Toxicol Sci 2012; 131:654-667.

20. Clarke G, Sandhu KV, Griffin BT, Dinan TG, Cryan JF, Hyland NP. Gut reactions: breaking down xenobiotic-microbiome interactions. Pharmacol Rev 2019; 71:198-224.

21. Selwyn FP, Cheng SL, Bammler TK, Prasad B, Vrana M, Klaassen $\mathrm{C}$, et al. Developmental regulation of drug-processing genes in livers of germ-free mice. Toxicol Sci 2015; 147:84-103.

22. Gonzalez FJ, Jiang C, Patterson AD. An intestinal microbiotafarnesoid $\mathrm{X}$ receptor axis modulates metabolic disease. Gastroenterology 2016; 151:845-859.

23. Zhernakova A, Kurilshikov A, Bonder MJ, Tigchelaar EF, Schirmer M, Vatanen T, et al. Population-based metagenomics analysis reveals markers for gut microbiome composition and diversity. Science 2016; 352:565-569.

24. Forbes JD, Van Domselaar G, Bernstein CN. The gut microbiota in immune-mediated inflammatory diseases. Front Microbio 2016; 7:1081.

25. Slingerland AE, Schwabkey Z, Wiesnoski DH, Jenq RR. Clinical evidence for the microbiome in inflammatory diseases. Front Immunol 2017; 8:400.

26. Sobhani I, Tap J, Roudot-Thoraval F, Roperch JP, Letulle S, Langella P, et al. Microbial dysbiosis in colorectal cancer (CRC) patients. PloS One 2011; 6:e16393.

27. Rogers G, Keating D, Young R, Wong M, Licinio J, Wesselingh $\mathrm{S}$. From gut dysbiosis to altered brain function and mental illness: mechanisms and pathways. Mol Psychiatry 2016; $21: 738$

28. Cani PD, Delzenne NM. Interplay between obesity and associated metabolic disorders: new insights into the gut microbiota. Curr Opin Pharmacol 2009; 9:737-743.

29. Li X, Watanabe K, Kimura I. Gut microbiota dysbiosis drives and implies novel therapeutic strategies for diabetes mellitus and related metabolic diseases. Front Immunol 2017; 8:1882.

30. Yang T, Santisteban MM, Rodriguez V, Li E, Ahmari N, Carvajal $\mathrm{JM}$, et al. Gut dysbiosis is linked to hypertension. Hypertension 2015; 65:1331-1340.

31. Maeda Y, Takeda K. Role of gut microbiota in rheumatoid arthritis. J Clin Med 2017; 6:60.

32. Lynch SV, Pedersen O. The human intestinal microbiome in health and disease. N Eng J Med 2016; 375:2369-2379.

33. Sokol H, Seksik P, Furet J, Firmesse O, Nion-Larmurier I, Beaugerie L, et al. Low counts of Faecalibacterium prausnitzii in colitis microbiota. Inflamm Bowel Dis 2009; 15:1183-1189.

34. Qiu X, Zhang M, Yang X, Hong N, Yu C. Faecalibacterium prausnitzii upregulates regulatory $\mathrm{T}$ cells and anti-inflammatory cytokines in treating TNBS-induced colitis. J Crohns Colitis 2013 7:e558-e568

35. Gkouskou K, Deligianni C, Tsatsanis C, Eliopoulos AG. The gut microbiota in mouse models of inflammatory bowel disease. Front Cell Infect Microbiol 2014; 4:28

36. Clemente JC, Manasson J, Scher JU. The role of the gut microbiome in systemic inflammatory disease. BMJ 2018; 360:j5145.

37. Schroeder BO, Bäckhed F. Signals from the gut microbiota to distant organs in physiology and disease. Nat Med 2016; 22:1079.

38. Swanson HI. Drug metabolism by the host and gut microbiota: a partnership or rivalry? Drug Metab Dispos 2015; 43:1499-1504.

39. Mullard A. Oncologists tap the microbiome in bid to improve immunotherapy outcomes. Nat Rev Drug Discov 2018; 17:153-155.

40. Viennois E, Gewirtz AT, Chassaing B. Chronic inflammatory diseases: Are we ready for microbiota-based dietary intervention? Cell Mol Gastroenterol Hepatol 2019; 8:61-71.

41. Alexander JL, Wilson ID, Teare J, Marchesi JR, Nicholson JK, Kinross JM. Gut microbiota modulation of chemotherapy efficacy and toxicity. Nat Rev Gastroenterol Hepatol 2017; 14:356-365.

42. Carbone C, Musumeci T, Pignatello R. Non-steroidal antiinflammatory drugs. In: Pignatello R, editor. Drug-Biomembrane Interaction Studies.The Application of Colorimetric Techniques. Cambridge: Woodhead Publishing; 2013. p 281-303,

43. Funk CD, FitzGerald GA. COX-2 inhibitors and cardiovascular risk. J Cardiovasc Pharmacol 2007; 50:470-479.

44. Wang D, DuBois RN. The role of COX-2 in intestinal inflammation and colorectal cancer. Oncogene 20101 29:781-788.

45. Dubois RN, Abramson SB, Crofford L, Gupta RA, Simon LS, A Van De Putte LB, Lipsky PE. Cyclooxygenase in biology and disease. FASEB J 1998; 12:1063-1073.

46. Ricciotti E, FitzGerald GA. Prostaglandins and inflammation. Arterioscler Thromb Vasc Biol 2011; 31:986-1000.

47. Mullins MN, Lana SE, Dernell WS, Ogilvie GK, Withrow SJ, Ehrhart E. Cyclooxygenase-2 expression in canine appendicular osteosarcomas. J Vet Intern Med 2004; 18:859-865.

48. Kang M, Martin A. Microbiome and colorectal cancer: Unraveling host-microbiota interactions in colitis-associated colorectal cancer development. Semin Immunol 2017;32:3-13. 
49. Lee HJ, Zhang H, Orlovich DA, Fawcett JP. The influence of probiotic treatment on sulfasalazine metabolism in rat. Xenobiotica 2012; 42:791-797.

50. Dethlefsen L, Relman DA. Incomplete recovery and individualized responses of the human distal gut microbiota to repeated antibiotic perturbation. Proc Natl Acad Sci U S A. 2011;108 Suppl 1:4554-61.

51. Mäkivuokko H, Tiihonen K, Tynkkynen S, Paulin L, Rautonen N. The effect of age and non-steroidal anti-inflammatory drugs on human intestinal microbiota composition. Brit J Nutr 2010; 103:227-234.

52. Rogers MA, Aronoff DM. The influence of non-steroidal antiinflammatory drugs on the gut microbiome. Clin Microbiol Infect 2016; 22:178. e1-178. e9.

53. Bokulich NA, Battaglia T, Aleman JO, Walker J, Blaser MJ, Holt PR. Celecoxib does not alter intestinal microbiome in a longitudinal diet-controlled study. Clin Microbiol Infect 2016; 22:464-465.

54. Walsh J, Griffin BT, Clarke G, Hyland NP. Drug-gut microbiota interactions: implications for neuropharmacology. Br J Pharmacol 2018; 175:4415-4429.

55. Clayton TA, Baker D, Lindon JC, Everett JR, Nicholson JK. Pharmacometabonomic identification of a significant hostmicrobiome metabolic interaction affecting human drug metabolism. Proc Natl Acad Sci U S A 2009; 106:14728-14733.
56. Tjalsma H, Boleij A, Marchesi JR, Dutilh BE. A bacterial driverpassenger model for colorectal cancer: beyond the usual suspects. Nat Rev Microbiol 2012; 10:575-582.

57. Zhu W, Winter MG, Byndloss MX, Spiga L, Duerkop BA, Hughes ER, et al. Precision editing of the gut microbiota ameliorates colitis. Nature 2018; 553:208-211.

58. Piard JC, Briandet R. Lactic acid bacteria biofilms: from their formation to their health and biotechnological potential. In: Mozzi F, Raya RR, Vignolo GM. Biotechnology of Lactic Acid Bacteria: Novel Applications: 2nd ed. West Sussex, UK: John Wiley \& Sons, Ltd; 2015. p. 341-361.

59. ElRakaiby M, Dutilh BE, Rizkallah MR, Boleij A, Cole JN, Aziz RK. Pharmacomicrobiomics: the impact of human microbiome variations on systems pharmacology and personalized therapeutics. OMICS 2014; 18:402-414.

60. Routy B, Le Chatelier E, Derosa L, Duong CP, Alou MT, Daillère R, et al. Gut microbiome influences efficacy of PD-1-based immunotherapy against epithelial tumors. Science 2018; 359:91-97.

61. Goossens N, Singal AG, King LY, Andersson KL, Fuchs BC, Besa C, et al. Cost-Effectiveness of Risk Score-Stratified Hepatocellular Carcinoma Screening in Patients with Cirrhosis. Clin Transl Gastroenterol 2017; 8:e101. 